\title{
La négociation administrée sur l'égalité professionnelle : entre respect de l'obligation et engagement formel
}

Administered Negotiation on Professional Equality: Between Compliance with

Legal Obligations and Formal Commitments

Denis Giordano et Guillaume Santoro

\section{(2) OpenEdition \\ Journals}

Édition électronique

URL : http://journals.openedition.org/travailemploi/9361

DOI : 10.4000/travailemploi.9361

ISSN : 1775-416X

Éditeur

DARES - Ministère du Travail

Édition imprimée

Date de publication : 2 novembre 2019

Pagination : 69-92

ISSN : 0224-4365

Référence électronique

Denis Giordano et Guillaume Santoro, « La négociation administrée sur l'égalité professionnelle : entre respect de l'obligation et engagement formel », Travail et Emploi [En ligne], 159 | 2019, mis en ligne le 01 janvier 2020, consulté le 07 décembre 2020. URL : http://journals.openedition.org/travailemploi/ 9361 ; DOI : https://doi.org/10.4000/travailemploi.9361 


\title{
La négociation administrée sur l'égalité professionnelle : entre respect de l'obligation et engagement formel"
}

\author{
Denis Giordano $^{* *}$, Guillaume Santoro ${ }^{* * *}$
}

La promotion de l'égalité professionnelle entre les femmes et les hommes constitue un enjeu prioritaire des politiques publiques. En France, le choix a été fait de privilégier la négociation collective pour instituer des mécanismes visant à résorber les inégalités présentes dans les entreprises. Plusieurs interventions législatives ont été nécessaires pour établir un dispositif juridique permettant de stimuler le dialogue social. Cet article propose d'analyser l'impact de ces réformes sur 282 textes signés par 200 entreprises de l'ancienne région Aquitaine. Nous soulignons comment ces évolutions législatives ont permis d'améliorer la négociation collective sur l'égalité professionnelle sans pour autant entraîner une amélioration des mesures ayant pour objectif de réduire les inégalités existantes en la matière dans les entreprises. Notre présentation va au-delà de l'analyse du seul respect des obligations légales pour apprécier les engagements formulés par les partenaires sociaux sur la période 2011-2015.

T e principe d'égalité constitue un droit fondamental (LANQUETIN et al., 2004), mais 1 l'établissement de l'égalité réelle au sein des entreprises rencontre de nombreux freins et nécessite la mise en œuvre d'actions spécifiques organisées dans le cadre de politiques publiques (PFEFFERKORN, 2002 ; BIT, 2012).

Le législateur a privilégié la négociation collective pour atteindre l'objectif d'égalité professionnelle entre les femmes et les hommes (JUNTER-LOISEAU, 1996)

\footnotetext{
* Ce travail a été réalisé dans le cadre du projet Egapro financé par la région Nouvelle-Aquitaine sous la direction de Guillaume Santoro au sein du laboratoire Comptrasec. Les auteurs tiennent à remercier Gautier Debruyne (Comptrasec) pour sa contribution au projet de recherche.

** Centre Maurice-Halbwachs (CNRS-ENS-EHESS) UMR 8097 Paris ; LCSP EA 7335 - université Paris 7 ; giordano.denis@gmail.com.

*** Comptrasec, UMR CNRS 5114 - université de Bordeaux ; guillaume.santoro@u-bordeaux.fr.
} 
dans le cadre de la loi « Roudy » en $1983^{1}$ et a consacré l'entreprise comme niveau pertinent de la négociation en raison de l'implication des acteurs et de leurs connaissances du terrain. Afin d'aboutir à une meilleure efficacité (Commaille, 2001), les pouvoirs publics ont opté pour ce que Guy Groux (2005) appelle « une action publique négociée », dont l'objectif est d'obliger les partenaires sociaux à engager des actions positives comme mesures de rattrapage (DAUPHIN, 2011). La loi « Génisson » de $2001^{2}$ a ainsi fixé le cadre et les outils que les différents acteurs doivent mobiliser pour construire des compromis permettant d'atteindre l'égalité entre les femmes et les hommes (Dugué, 2005 ; LANQueTIN, 2017).

L'ensemble des dispositions vise à encadrer et à encourager le dialogue social pour faire évoluer les mentalités et les pratiques au sein des entreprises (JUNTER, 2004 ; Milewski, SÉNAC, 2014). Le dispositif initial de négociation sur l'égalité professionnelle n'ayant pas eu les effets escomptés, le législateur est intervenu à de multiples reprises au cours des quinze dernières années pour le compléter afin de le rendre plus efficient (MINÉ, 2017). En 2010, la loi portant réforme des retraites ${ }^{3}$ a introduit de nouvelles mesures imposant aux entreprises d'au moins 50 salarié.es d'être couvertes par un accord collectif ou, à défaut, par un plan d'action fixant des objectifs de progression, des actions permettant de les atteindre et des indicateurs chiffrés sur au moins deux ou trois domaines d'action (selon que l'entreprise compte plus ou moins de 300 salarié.es $)^{4}$, sous peine de se voir appliquer une sanction pécuniaire. Ce dispositif a été renforcé en $2012^{5}$, avec l'ajout d'un domaine d'action à aborder dans les textes et l'obligation faite aux entreprises de négocier sur la rémunération.

L'État a durci le cadre des négociations dans un double but : accroître le taux des entreprises couvertes par un accord collectif et améliorer le contenu des accords et des plans d'action. Si le nombre de textes a progressé, l'augmentation des contraintes visant à stimuler la négociation collective entre les partenaires sociaux n'a pas forcément entraîné une amélioration du contenu des textes, car la négociation rencontre de nombreux freins. En effet, en dépit de l'obligation légale à laquelle sont soumises les entreprises, la thématique de l'égalité professionnelle n'est toujours pas considérée comme prioritaire au sein des négociations collectives d'entreprise. Les employeurs la voient comme une contrainte supplémentaire (LAUFER, 2009), régulièrement supplantée par les négociations sur les salaires, le temps de travail et l'emploi (MinISTĖRE DU TRAVAIL, 2018). La mobilisation des représentants syndicaux n'est pas non plus optimale, car le sujet est clivant, les revendications des salarié.es, femmes et hommes,

1. Loi du 13 juillet 1983 portant modification du Code du travail et du Code pénal en ce qui concerne l'égalité professionnelle entre les hommes et les femmes.

2. Loi du 9 mai 2001 relative à l'égalité professionnelle entre les femmes et les hommes.

3. Loi du 9 novembre 2010 portant réforme des retraites.

4. Sur la période étudiée, le dispositif juridique prévoyait huit domaines d'action : embauche, formation, promotion professionnelle, qualification, classification, conditions de travail, rémunération effective, articulation entre l'activité professionnelle et la vie personnelle et familiale. La loi du 4 août 2014 pour l'égalité réelle entre les femmes et les hommes a rajouté un neuvième domaine : la santé et la sécurité au travail, qui n'a pas été pris en compte dans le cadre de cette étude.

5. Décret du 18 décembre 2012 relatif à la mise en œuvre des obligations des entreprises pour l'égalité professionnelle entre les femmes et les hommes. 
sont peu nombreuses et les femmes sont sous-représentées dans les instances de négociations (SiLVERA, 2006 ; CRISTOFALO, 2014). Enfin, certains partenaires sociaux ont peut-être parfois renoncé à engager des négociations, leurs faibles connaissances juridiques (SANTORO, 2016) ne leur permettant pas de se confronter à la multiplication des thématiques à aborder dans les négociations, à leur complexification en raison de leur caractère de plus en plus technique (MIAS, 2014) et aux renforcements successifs des contraintes du dispositif sur l'égalité professionnelle.

Les multiples réformes et les difficultés à faire évoluer significativement l'égalité professionnelle font l'objet de nombreux débats et critiques (MARUANI, 2013 ; LAUFER, 2014 ; Le Quentrec, BACOU, 2017). Les précédentes recherches menées sur la négociation relative à l'égalité professionnelle depuis l'introduction d'une sanction financière pour défaut de texte font état d'un respect global du dispositif législatif, mais relèvent de nombreuses imprécisions dans son application en raison d'une mauvaise connaissance des dispositions légales de la part des entreprises (BRUNET, Dumas, 2012 ; BUCHER et al., 2015 ; CONSEIL SUPÉRIEUR DE L'ÉGALITÉ PROFESSIONNELLE ENTRE LES FEMMES ET LES HOMMES, 2014). La persistance des inégalités dans l'emploi nous conduit à nous interroger sur le modèle même du dispositif légal adopté et sur les résultats de la négociation administrée ${ }^{6}$ sur l'égalité professionnelle. L'objectif de notre démarche est d'appréhender les évolutions consécutives à la réforme de 2010 en portant une attention particulière à la continuité des engagements issus des renégociations. C'est pourquoi il nous a semblé instructif de réaliser une analyse détaillée des textes adoptés par les entreprises afin d'évaluer la mise en œuvre du dispositif, l'incidence des différentes modifications législatives dont il a fait l'objet, et de comprendre les freins à son application (encadré 1).

Cette contribution s'inscrit dans le cadre des analyses actuelles des politiques publiques (DELPEUCH et al., 2014 ; HASSENTEUfEL, 2011) qui s'intéressent particulièrement à leur mise en œuvre concrète (COMMAILlE, DURAN, 2009 ; PADIOLEAU, 1982). Nous nous concentrons ici sur les modalités d'application « en action » (JOBERT, MuLLER, 1987) des engagements sur l'égalité professionnelle qui, dans le cadre de la négociation obligatoire, s'inscrivent au niveau de la politique d'entreprise. Nous considérons donc les écarts à la règle de droit non comme une anomalie ou un signe de faillite du dispositif législatif, mais comme une conséquence de l'utilisation qui peut en être faite (LASCOUMES, LE BOURHIS, 1996) par les acteurs mobilisés lors des négociations. De tels écarts à la règle de droit se retrouvent également dans les pratiques des contrôleurs de l'administration qui adoptent parfois une approche pragmatique face aux faiblesses et aux « erreurs » des textes de négociation (WELLER, 2007). Notre objectif est de faire un état des lieux de l'application du dispositif législatif en soulignant les résistances et les évolutions ayant eu lieu en matière d'égalité professionnelle dans un contexte de « foisonnement législatif» (LAUFER, SILVERA, 2017).

6. La « négociation administrée » désigne les dispositifs mis en place par l'État pour inciter les entreprises à négocier des accords ou des plans d'action sur des sujets particuliers. Il s'agit d'injonctions à négocier, avec des prescriptions spécifiques sur des thèmes obligatoires, sous peine de sanctions pécuniaires. 


\section{ENCADRÉ 1}

\section{Méthodologie de recherche}

Le dispositif juridique relatif à l'égalité professionnelle adopté en 2010 et entré en vigueur le $1^{\text {er }}$ janvier 2012 a été renforcé en décembre de la même année. C'est pourquoi nous avons souhaité étudier les textes signés par les entreprises de l'ancienne région Aquitaine $^{1}$ dans le cadre de la négociation collective obligatoire sur l'égalité professionnelle, déposés à la Direction régionale des entreprises, de la concurrence, de la consommation, du travail et de l'emploi (Direccte ${ }^{2}$ ) Aquitaine au cours de la période allant de janvier 2011 à décembre 2015, afin de pouvoir suivre l'évolution de leur contenu et de leur renouvellement au regard des différentes réformes législatives mises en œuvre au cours de cette période. Par « textes », nous entendons les accords collectifs négociés et les plans d'action adoptés unilatéralement par les employeurs en cas d'échec des négociations ${ }^{3}$.

Notre corpus a été constitué à partir de 1240 textes signés entre 2011 et 2015 par 779 entreprises de l'ancienne région Aquitaine assujetties à l'obligation de négocier (entreprises de 50 salarié.es et plus), contrôlés par l'administration et enregistrés dans le logiciel D@ccord de la Direccte. L'ensemble des textes ne pouvant être analysé, nous avons constitué deux échantillons aléatoires simples de 100 entreprises chacun, en retenant l'effectif de l'entreprise comme catégorie de référence afin de prendre en compte le dispositif juridique qui différencie les entreprises de plus et de moins de 300 salarié.es. Le premier échantillon a été construit à partir des 671 entreprises de 50 à 299 salarié.es (ciaprès T. -300), et le second à partir des 108 entreprises de 300 salarié.es et plus (ci-après T. 300+), ce qui représente un échantillon pratiquement exhaustif. L'analyse a porté sur 140 textes pour les T. -300 et 142 pour les T. $300+{ }^{4}$.

Le choix de considérer de manière égale les petites (T. -300) et les grandes entreprises (T. 300+) a pour objectif d'évaluer, avec des effectifs identiques (100 entreprises pour chaque échantillon), les analogies et les différences d'engagement sur l'égalité professionnelle dans des contextes organisationnels divers, et de détailler l'impact du dispositif législatif différencié selon la taille.

Les 779 entreprises sont situées dans les cinq départements de l'ancienne région Aquitaine et sont représentatives de son tissu économique. Les entreprises de l'échantillon T. -300 sont réparties sur l'ensemble de la région, alors que celles de 300 salarié.es et plus sont, pour $69 \%$ d'entre elles, localisées dans le département de la Gironde et relèvent plus particulièrement du tissu économique de la métropole bordelaise. Comme les entreprises de même effectif au niveau national ${ }^{5}$, les entreprises sélectionnées exercent leur activité

1. Notre base de données initiale comprend l'ensemble des textes des entreprises des cinq départements de l'ancienne région Aquitaine (Gironde, Dordogne, Landes, Pyrénées-Atlantiques et Lot-et-Garonne).

2. La Direccte est un service de l'État chargé de piloter les politiques publiques au niveau régional, notamment en contrôlant les textes signés par les entreprises dans le cadre de leur obligation de négocier sur l'égalité professionnelle entre les femmes et les hommes.

3. L'analyse propose donc un panorama des seuls textes signés par les entreprises ayant négocié en Aquitaine et ne concerne pas les entreprises n'ayant pas mis en œuvre de négociations.

4. Enfin, nous avons mené un entretien avec un contrôleur de la Direccte qui nous a précisé les modalités de contrôle définies dans les instructions de la Direction générale du travail (DGT).

5. Selon la nomenclature d'activités française (NAF) à dix postes. 
La négociation administrée sur l'égalité professionnelle : entre respect de l'obligation et engagement formel

principalement dans trois secteurs : le commerce, les transports et l'hébergement $(27,1 \%)$, l'industrie $(24,3 \%)$ et la construction $(20,9 \%)$.

Nous avons choisi le texte de négociation produit par l'entreprise comme unité d'analyse. Nous nous sommes concentrés sur le processus de négociation et sur l'argumentation développée par les entreprises dans les différents domaines d'action, mais également sur l'évolution des textes au cours de la période de référence au regard des modifications législatives intervenues (cf. supra). Nous avons rencontré des difficultés d'analyse en raison de la complexité des accords et des plans d'action rédigés par des acteurs différents, dans des contextes variés selon les secteurs d'activité et faisant de multiples références aux normes juridiques et aux rhétoriques managériales (AMINTAS, JUNTER, 2009). C'est pourquoi, afin de systématiser les différents éléments cités, nous les avons archivés à l'aide d'une grille de saisie auto-compilée en les classant par thématiques ${ }^{6}$.

Nous nous sommes particulièrement intéressés, d'une part, aux informations obligatoires découlant du dispositif juridique et, d'autre part, aux éléments détaillant les engagements des parties. Au-delà des obligations légales, aucun formalisme n'était imposé pour la rédaction des textes, si bien que le nombre d'informations varie beaucoup d'un texte à l'autre. Notre corpus relatif aux informations «non obligatoires » est par conséquent marqué par la présence de nombreuses « absences », car les textes sont très hétéroclites et, pour certains, très pauvres. Nous avons également constaté que des indications formelles étaient fréquemment manquantes, particulièrement celles relatives au processus de négociation et au texte précédent ${ }^{7}$. Nous nous sommes de plus concentrés sur les 2986 actions souscrites dans les textes en constituant une base de données dédiée. Afin de clarifier le contenu des textes, nous avons développé une analyse sur l'ensemble des actions en les classant par catégories. L'extrême hétérogénéité des actions et la difficulté à systématiser leurs caractéristiques ont rendu la tâche complexe. Nous avons donc élaboré un protocole ${ }^{8}$ (cf. infra) pour déterminer la catégorie de chaque action en fonction de la description de la thématique abordée 9

Pour résumer, nous avons constitué une base de données principale (en différenciant les deux échantillons) et deux bases complémentaires dédiées aux actions et aux renégociations des textes.

6. Les informations sont classées de façon à présenter la structure du texte, le processus de négociation, les acteurs engagés, le préambule, les références juridiques, le contenu détaillé domaine par domaine et action par action, les différentes dispositions, etc.

7. Partant de l'hypothèse d'une structuration très articulée des textes, nous avons retenu plus de 700 informations. Nous n'avons pu ensuite en exploiter qu'une partie très réduite, car toutes ne trouvaient pas de consistance dans l'ensemble des textes.

8. Le protocole indique les critères pour différencier les actions avec une liste d'exemples pour chaque catégorie. Pour mieux attribuer les actions aux différentes catégories, nous les avons décrites par des mots-clés que nous avions préalablement définis.

9. Nous ne nous sommes intéressés ici ni à la finalité de l'action ni à la finalité pour les salarié.es. 
Nous souhaitons contribuer au débat en confrontant les contradictions entre le perfectible respect des critères législatifs et les résultats mitigés, dans les faits, de l'application de textes parfois étiquetés « coquilles vides », ou " empty shells », expression déjà employée pour indiquer l'issue de négociations sur l'égalité professionnelle (ChARPENEl et al., 2017 ; HoQue, NoOn, 2004). Dans une première partie, nous exposerons dans quelle mesure les accords collectifs et les plans d'action respectent le nouveau dispositif. Puis, dans une seconde partie, nous présenterons une analyse de l'engagement des acteurs sur la thématique de l'égalité professionnelle en décrivant les mesures souscrites.

\section{Un cadre législatif insuffisamment contraignant}

L'obligation de négocier sur l'égalité professionnelle a été encadrée par un dispositif législatif spécifique accompagné d'un protocole de contrôle par l'administration. L'étude des textes déposés auprès de la Direccte Aquitaine nous a permis d'analyser l'application du dispositif juridique et de déceler les limites des mesures introduites par le législateur sur le plan de l'engagement des négociations, du contenu des textes et au regard du contrôle réalisé par l'administration.

\section{Une négociation accrue par un dispositif plus coercitif}

Devant la persistance des inégalités et le faible résultat des négociations (LAUFER, SILVERA, 2005 ; RABIER, 2009), principalement dus à une mobilisation insuffisante des acteurs, le législateur est intervenu en 2010 dans le cadre de la loi portant réforme des retraites pour contraindre les employeurs à négocier ou à prendre des dispositions unilatérales sur la thématique de l'égalité professionnelle. La loi fixe désormais un cadre de négociation avec une pénalité financière pour les entreprises de 50 salariées et plus qui ne seraient pas couvertes par un accord collectif ou un plan d'action relatif à l'égalité professionnelle. Il s'agit d'une obligation de moyens qui se limite à exiger l'engagement par l'employeur d'une négociation annuelle avec les représentants syndicaux $^{7}$. Il n'y a pas d'obligation d'aboutir à un accord puisque l'employeur peut signer un plan d'action unilatéral en cas d'échec des négociations. L'efficacité du dispositif repose sur une sanction pécuniaire appliquée en cas de manquement à l'obligation annuelle de négocier après vérification des contrôleurs de la Direccte.

L'objectif du nouveau dispositif a porté ses fruits, comme le démontre la très forte augmentation du nombre de textes signés dès 2012, année de l'entrée en vigueur de la loi. Le nombre de textes a été multiplié par cinq dans notre échantillon et s'est stabilisé

7. Pendant la durée de l'étude, l'obligation était annuelle et le demeurait dans le cas de la signature d'un plan d'action, mais devenait triennale en cas de signature d'un accord. Depuis l'ordonnance du 22 septembre 2017, il y a obligation de négocier au moins une fois tous les quatre ans. L'obligation ne devient annuelle qu'en l'absence de négociations. Il y a donc eu un inversement de la durée des obligations. 
La négociation administrée sur l'égalité professionnelle : entre respect de l'obligation et engagement formel

à ce niveau pendant toute la durée de la période analysée : le caractère coercitif du dispositif a donc permis de stimuler les partenaires sociaux, ces résultats étant toutefois à relativiser, car ils concernent les seuls textes déposés auprès de la Direccte ${ }^{8}$.

L'augmentation du nombre de textes signés - et donc des négociations engagées conduit à s'interroger sur l'impact du dispositif sur la nature des textes déposés. Si l'on regarde la nature juridique des textes (graphique), on peut souligner la forte présence d'accords dans l'échantillon de textes des entreprises de grande taille, et ce pour toutes les années prises en compte (cette part n'est jamais inférieure à 68,8\%). Au sein des plus petites entreprises, la part des accords est plus faible et plus fluctuante, oscillant entre un tiers et deux tiers des textes 9 .

\section{GRAPHIQUE - Part des accords parmi les textes, selon l'année}

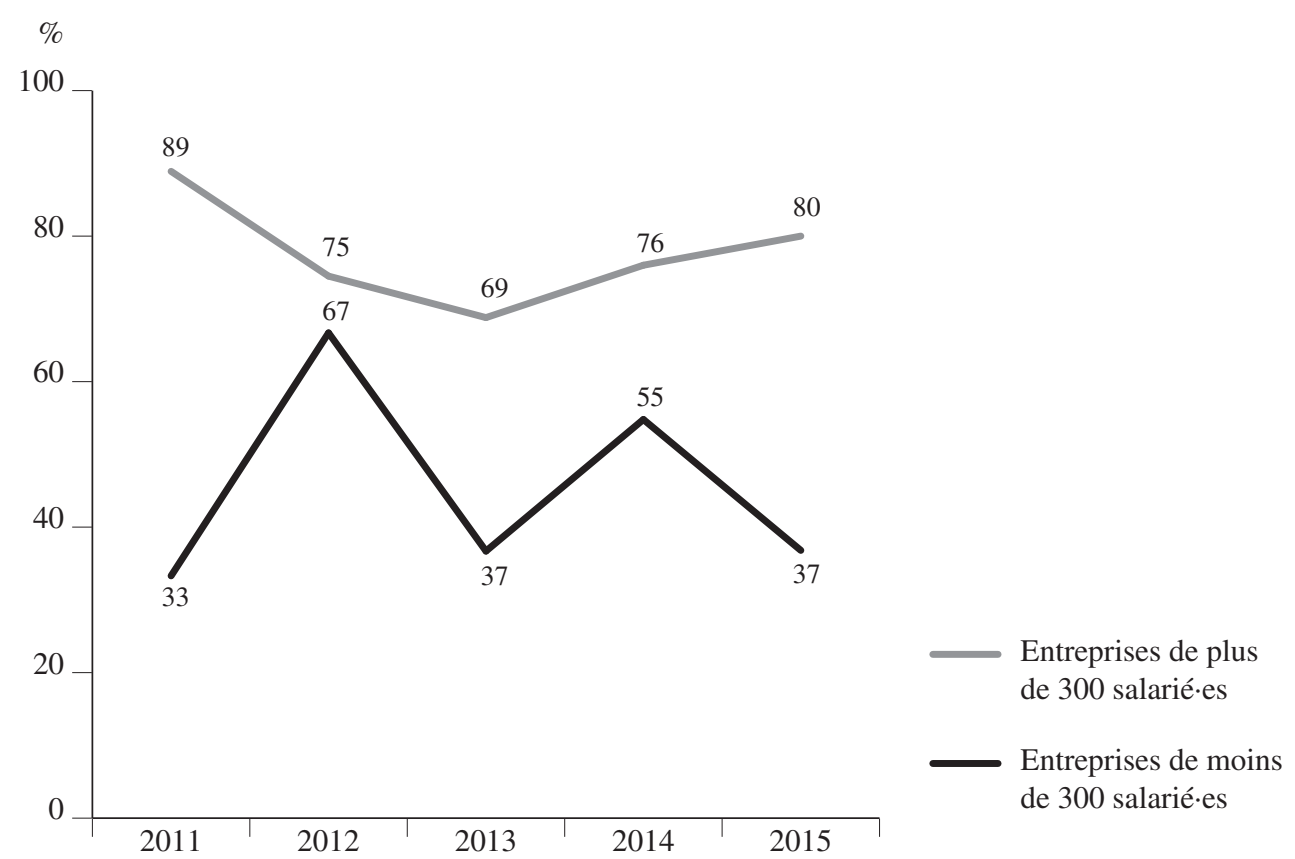

Lecture : parmi les textes des T. -300 déposés en 2011, $89 \%$ sont des accords.

Champ : 282 textes négociés et signés entre 2011 et 2015 dans l'ancienne région Aquitaine (140 pour les T. -300 et 142 pour les T. $300+$ ). Source : données du projet Egapro.

8. Seules $54 \%$ des entreprises d'Aquitaine sont couvertes par un texte fin 2015 selon le relevé mensuel de la Direccte Aquitaine de novembre 2015.

9. Parmi les T. $300+$, les accords sont beaucoup plus nombreux que les plans d'action signés unilatéralement (107 contre 35), alors que parmi les T. -300 , les plans d'action sont plus nombreux (74 contre 66). 
Comme cela a été souligné dans d'autres analyses ${ }^{10}$, la présence d'une majorité d'accords collectifs démontre que la nature plus contraignante du dispositif a permis d'améliorer l'engagement et d'assurer la réussite des négociations. Même si $39 \%$ des textes demeurent des plans d'action - ce qui révèle la faiblesse persistante du dispositif -, on peut considérer que cela constitue un début de prise en compte de la thématique de l'égalité professionnelle au sein des entreprises.

Il y a plusieurs explications à la non-concrétisation des négociations et au fait que les employeurs aient pris des engagements unilatéraux. D'abord, la possibilité laissée à l'employeur de signer un plan d'action unilatéral, à défaut d'accord collectif, ne favorise pas le développement de la négociation collective. Le législateur est d'ailleurs intervenu en $2012^{11}$ pour limiter cette pratique, en exigeant que les entreprises de 300 salarié es et plus signent un procès-verbal de désaccord en cas d'échec des négociations. Celui-ci doit contenir les propositions respectives des parties et les mesures que l'employeur entend appliquer unilatéralement. Enfin, des causes inhérentes à la taille des entreprises ne facilitent pas la négociation. Il en va ainsi des petites entreprises, qu'un effectif légèrement supérieur à 50 salarié·es oblige à négocier alors qu'elles n'ont pas la structure organisationnelle des grandes entreprises, avec des membres du personnel dédiés à ces questions (ressources humaines, délégués syndicaux et représentants du personnel), qui leur permettraient d'avoir la connaissance juridique nécessaire pour mener à bien l'ensemble des négociations obligatoires auxquelles elles sont soumises. Cette distinction selon l'effectif des entreprises pointe les écueils auxquels sont confrontées celles dont l'effectif dépasse tout juste le seuil de 50 salariées. Le dispositif étant assez similaire pour l'ensemble des entreprises, il est par conséquent trop contraignant pour les plus petites d'entre elles. Les obstacles qu'elles rencontrent en raison de leur petite taille n'ont pas assez été pris en compte par le législateur.

L'originalité de notre recherche réside dans la période analysée puisque nous avons fait le choix d'étudier les textes déposés entre 2011 et 2015 pour saisir l'évolution quantitative et qualitative des accords et des plans d'action lors des négociations et des renégociations, au regard des modifications législatives successives intervenues au cours de cette période. Pour traiter les différentes périodes couvertes par un texte, nous avons constitué quatre classes de taux de couverture ${ }^{12}$ selon la durée pendant laquelle les entreprises sont couvertes, à savoir pendant la totalité des cinq années ; pendant plus de la moitié de cette période ${ }^{13}$; pendant moins de la moitié ; et enfin une durée nulle lorsqu'elles sont sans couverture (tableau 1).

10. Dans le rapport de BUCHER et al. (2015), il y a une plus forte présence d'accords (84 \%). Le rapport de la DIRECCTE PACA (2013) a également relevé 59 accords et 7 plans.

11. Loi du 26 octobre 2012 portant création des emplois d'avenir.

12. Par « couverture », nous entendons le nombre de mois pendant lesquels un texte sur l'égalité professionnelle est présent dans notre corpus et en cours de validité sur la période 2011-2015.

13. C'est-à-dire plus de deux ans et six mois. 
TABLEAU 1 - Nombre d'entreprises couvertes par un texte relatif à l'égalité professionnelle en fonction de la durée de couverture et de la taille de l'entreprise (2011-2015)

\begin{tabular}{lcc}
\hline Durée de couverture & Entreprises - 300 salarié·es & Entreprises 300+ salarié·es \\
\hline Aucune & 2 & 1 \\
Moins de la moitié de la période & 26 & 11 \\
Moitié de la période et plus & 52 & 51 \\
Totalité de la période & 20 & 37 \\
\hline Effectif total & $\mathbf{1 0 0}$ & $\mathbf{1 0 0}$ \\
\hline
\end{tabular}

Lecture : parmi les entreprises de moins de 300 salarié·es, 2 ne sont couvertes par aucun texte relatif à l'égalité professionnelle sur la période 2011-2015.

Champ : 200 entreprises ayant négocié et signé des textes entre 2011 et 2015 dans l'ancienne région Aquitaine (140 pour les T. -300 et 142 pour les T. $300+$ ).

Source : données du projet Egapro.

Le dispositif juridique introduit en 2012 a permis d'améliorer la négociation et la renégociation d'accords ou de plans d'action, avec cependant une différence de taux de couverture selon l'effectif des entreprises, que fait apparaître l'analyse des données. Ainsi, $37 \%$ des entreprises de 300 salarié.es et plus ont un taux de couverture sur toute la période de cinq années, mais c'est le cas de seulement $20 \%$ de celles de moins de 300 salarié.es. Le fait que les entreprises de plus grande taille respectent mieux la temporalité de l'obligation de couverture peut de nouveau s'expliquer par leurs moyens plus conséquents en matière de ressources humaines, qui leur permettent de respecter les prescriptions temporelles des obligations de négocier. La moitié des entreprises ont un taux de couverture courant sur plus de la moitié de la période, sans pour autant que ce soit tout au long de celle-ci. L'importance de cette part s'explique par les quelques mois de carence dus à des retards dans le dépôt des textes auprès de la Direccte. Au-delà de ces courtes périodes d'absences de couverture, nous avons décelé pour l'ensemble des entreprises des déficiences beaucoup plus grandes, d'une durée d'un an ou plus, qui semblent plutôt correspondre à un désintérêt de la négociation sur la thématique de l'égalité professionnelle ; les parties étant plus concentrées sur les négociations relatives aux salaires et au temps de travail et les employeurs demeurant relativement indifférents aux risques d'une sanction financière.

Concernant les renégociations de textes, nous avons relevé 69 entreprises ayant renégocié plusieurs textes, avec une prédominance d'accords collectifs (sur l'ensemble de l'échantillon, $61 \%$ des textes renégociés étaient des accords contre $39 \%$ de plans d'action). Nous nous sommes alors demandé si les renégociations entraînaient ou non une réappropriation des textes par les négociateurs pour aller au-delà des prescriptions légales. La réponse est négative. En effet, alors que les partenaires sociaux s'étaient engagés au-delà des obligations minimales lors des premières négociations, ils se sont limités à actualiser les textes au regard des obligations légales lors des renégociations.

Quant à la forme juridique des textes, nous constatons que seulement dix entreprises l'ont modifiée lors des renégociations, neuf d'entre elles ayant signé un accord après avoir adopté un plan d'action lors de la négociation précédente. Le passage du 
plan à l'accord peut s'expliquer par le fait que l'employeur a intérêt à augmenter la durée de validité du texte d'un an à trois ans. Mais comme ce choix s'accompagne de l'obligation de négocier avec les représentants du personnel, cela suppose de faire certaines concessions pour aboutir à un accord. Une seule entreprise a fait le choix inverse, en optant pour un plan d'action après avoir préalablement négocié un accord collectif. Notre analyse montre que le choix offert aux employeurs entre la signature d'un accord ou d'un plan d'action n'a pas eu pour effet de les engager vers une augmentation des engagements unilatéraux, qui était une voie plus facile.

\section{Un dispositif formaliste pour encadrer le contenu des textes}

Outre le cadrage sur l'engagement à négocier, le législateur a souhaité orienter le contenu des textes en listant des thématiques spécifiques pour la négociation et en enjoignant les entreprises à les articuler.

Les parties impliquées dans la négociation doivent choisir un nombre minimal de domaines d'action ${ }^{14}$ au sein de la liste prévue, selon l'effectif de l'entreprise. Le nombre minimal a été augmenté en 2012 par rapport au dispositif initial, passant de deux à trois pour les entreprises de moins de 300 salarié.es, et de trois à quatre pour celles de 300 salarié.es et plus.

L'observation du nombre moyen de domaines d'action inclus dans les textes signés avant la réforme de décembre 2012 ne permet pas de déceler de différences importantes selon l'effectif de salarié·es (tableau 2). Les entreprises de moins de 300 salarié·es ont choisi en moyenne 3,4 domaines d'action (pour une obligation de 2) alors que les entreprises de 300 salarié.es et plus atteignent une moyenne de 3,6 domaines d'action (pour une obligation de 3 ). Ces chiffres montrent le respect de l'obligation par l'ensemble des entreprises, quel que soit leur effectif. Après la réforme, les entreprises ont observé un strict respect du nombre de domaines obligatoires, ce qui signifie que l'engagement supplémentaire a été réduit. Ainsi, les entreprises de moins de 300 salarié·es ont rempli leur obligation de choisir trois domaines d'action en conservant une moyenne de

TABLEAU 2 - Nombre moyen de domaines d'action choisis par texte selon la taille de l'entreprise et la période

\begin{tabular}{lcc|cc|cc|ccc}
\hline & \multicolumn{3}{c}{ T. -300 } & \multicolumn{4}{c}{ T. 300+ } \\
\hline Avant 2013 & $\mathbf{N}$ & $\mathbf{2 0 1 3 - 2 0 1 5}$ & $\mathbf{N}$ & Avant 2013 & $\mathbf{N}$ & $\mathbf{2 0 1 3 - 2 0 1 5}$ & $\mathbf{N}$ \\
\hline Plans & 3,1 & 12 & 3,2 & 63 & 3,5 & 14 & 4,1 & 21 \\
Accords & 3,6 & 18 & 3,6 & 47 & 3,7 & 46 & 4,1 & 61 \\
\hline Textes & $\mathbf{3 , 4}$ & $\mathbf{3 0}$ & $\mathbf{3 , 4}$ & $\mathbf{1 1 0}$ & $\mathbf{3 , 6}$ & $\mathbf{6 0}$ & $\mathbf{4 , 1}$ & $\mathbf{8 2}$ \\
\hline
\end{tabular}

Lecture : avant 2013, pour un effectif de 12 plans signés dans des entreprises de moins de 300 salarié.es, le nombre moyen des domaines d'action est de 3,4.

Champ : 282 textes négociés et signés entre 2011 et 2015 dans l'ancienne région Aquitaine (140 pour les T. - 300 et 142 pour les T. 300+). Source : données du projet Egapro.

14. Les domaines ont été détaillés dans la note 4 (cf. supra). 
3,4 domaines par texte. Celles de 300 salarié.es et plus se sont limitées à leur obligation en adoptant une moyenne de 4,1 domaines d'action par texte.

Les modifications législatives ont donc été largement suivies et sont susceptibles d'apporter des évolutions dans les pratiques de négociation collective. Toutefois, l'entrée en vigueur d'une loi plus contraignante ne se traduit pas par un changement immédiat de comportement, mais déclenche le plus souvent une dynamique qu'il est possible d'évaluer et apprécier au fil du temps.

En 2012, le décret d'application ne s'est pas limité à augmenter le nombre de domaines d'action, il a également imposé que la « rémunération » soit présente dans les textes. Or, avant d'être obligatoire, ce domaine d'action était peu choisi par les parties impliquées dans les négociations ${ }^{15}$, en raison de l'obligation annuelle de négocier sur les salaires et du coût financier de dispositions sur la rémunération effective dans les accords d'égalité professionnelle. Une fois le domaine d'action devenu obligatoire, les entreprises se sont adaptées et respectent le dispositif dans $90 \%$ des textes (tableau 3).

TABLEAU 3 - Présence du domaine d'action « rémunération » dans les textes selon les années

\begin{tabular}{lcccccc}
\hline & $\mathbf{2 0 1 1}$ & $\mathbf{2 0 1 2}$ & $\mathbf{2 0 1 3}$ & $\mathbf{2 0 1 4}$ & $\mathbf{2 0 1 5}$ & $\mathbf{N}$ \\
\hline T. -300 & $33 \%$ & $33 \%$ & $86 \%$ & $93 \%$ & $87 \%$ & 140 \\
T. $300+$ & $44 \%$ & $43 \%$ & $78 \%$ & $92 \%$ & $91 \%$ & 142 \\
\hline Effectif & 15 & 75 & 62 & 67 & 63 & 282 \\
\hline
\end{tabular}

Lecture : en 2011, le domaine d'action « rémunération » est présent dans $33 \%$ des textes des entreprises de moins de 300 salarié.es. Champ : 282 textes négociés et signés entre 2011 et 2015 dans l'ancienne région Aquitaine (140 pour les T. -300 et 142 pour les T. 300+). Source : données du projet Egapro.

Puisque la réforme de 2012 a permis le respect des nouvelles obligations, ne serait-il pas pertinent d'exiger des entreprises qu'elles développent l'ensemble des domaines d'action sans se limiter à un nombre minimal ? L'exemple précité de la rémunération montre en effet que les entreprises ont respecté l'obligation dès la mise en place du dispositif. Cependant, le contenu introduit par les signataires des textes invite à en relativiser l'impact. Comme nous le verrons infra, le respect de l'obligation n'est en effet pas synonyme de mesures à même de résorber les inégalités entre les femmes et les hommes.

L'obligation qu'ont les entreprises d'argumenter sur le contenu des textes en choisissant des domaines d'action particuliers exige que les parties impliquées dans la négociation décrivent les mesures pour mener à bien les changements : elles doivent donc fixer des objectifs de progression, déterminer des actions permettant de les atteindre et des indicateurs chiffrés permettant de les suivre. Ces trois éléments font l'objet d'une vérification par les contrôleurs de la Direccte lors de l'enregistrement du texte. 
L'analyse menée sur notre échantillon montre que l'obligation de caractériser les démarches est assez largement respectée pour les objectifs et les actions, mais dans une moindre mesure pour les indicateurs. En effet, $84 \%$ des T. -300 et $97 \%$ des T. 300+ ont intégré au moins un objectif par domaine. L'introduction d'actions est également bien suivie par les entreprises, et ce quelle que soit leur taille ${ }^{16}$. En revanche, seuls $63 \%$ des textes des T. -300 et $77 \%$ des T. 300+ ont fixé un indicateur par domaine d'action. Or les indicateurs chiffrés constituent des éléments importants, car ils permettent de suivre les objectifs et les actions pendant la durée d'exécution du texte, d'évaluer leurs applications et peuvent servir de base dans une renégociation.

\section{Un dispositif contraignant appliqué sous le contrôle bienveillant de l'administration}

La lecture des informations relatives à la mise en œuvre des obligations concernant l'articulation des domaines d'action doit être réalisée à la lumière du contrôle exercé par l'administration. La sanction pécuniaire est la mesure principale du dispositif ayant une incidence sur la négociation. Cependant, cette sanction n'est pas immédiate et n'est que l'aboutissement d'une procédure initiée par les contrôleurs de la Direccte.

Les contrôleurs vérifient la présence ou non d'une réponse au dispositif législatif, puis se rapprochent des entreprises assujetties à l'obligation de négocier sur l'égalité professionnelle pour engager une discussion avec elles. Dès lors qu'un manquement aux obligations légales est constaté, il appartient à l'administration de prendre les mesures adaptées. Le législateur a prévu que l'employeur puisse être mis en demeure de remédier à la situation constatée dans un délai de six mois. Or les informations communiquées par l'administration de la Direccte Nouvelle-Aquitaine font état des limites du contrôle exercé puisque les contrôleurs ne disposent pas de fichiers à jour des entreprises assujetties à l'obligation de négocier. Ainsi, l'implantation de nouvelles entreprises ou les changements de seuil de 50 et 300 salarié.es ne leur sont pas immédiatement communiqués. De plus, les contrôleurs sont en nombre insuffisant. L'obligation d'être couvert par un accord ou un plan d'action et le délai de mise en demeure trouvent ainsi des limites dans leur application et remettent en cause l'efficacité du volet coercitif du dispositif.

Comme précisé, la période étudiée est particulière, car elle couvre le début de la mise en œuvre du dispositif. L'ensemble des obligations n'a pas pu être acquitté parfaitement les premières années. Cette situation avait été envisagée par l'administration, puisque l'instruction de la Direction générale du travail (DGT) de 2013 relative à la stratégie du contrôle de la mise en œuvre des obligations prévoyait un contrôle strict, mais également un plan d'action plus large offrant un « accès à des démarches d'appropriation et d'accompagnement ». Cette instruction avait également prévu que le contrôle soit d'abord réalisé auprès des entreprises de plus de 1000 salarié·es, puis

16. $93 \%$ des T. -300 et $94 \%$ des T. 300+ ont introduit une action pour chaque domaine d'action choisi. 
auprès de celles de plus de 300 salarié.es et, enfin, de celles de moins de 300 salarié.es, le tout étalé sur l'année 2013 et le début de l'année 2014. De telles directives ont pu avoir une incidence sur les négociations collectives lors des premières années du dispositif et ainsi engendrer des différences selon la taille des entreprises.

Cet accompagnement explique également que les décisions prises à la suite des contrôles ne soient pas toujours très contraignantes, car les contrôleurs doivent prendre en compte l'environnement spécifique de chaque entreprise ${ }^{17}$. Ainsi, lorsqu'il manque un seul objectif ou un seul indicateur dans un plan d'action qui devra être renégocié un an plus tard, le contrôle est moins pressant que lorsqu'il manque une action dans un accord collectif conclu pour une durée de trois ans. Précisons que si l'employeur peut modifier seul un plan d'action, modifier un accord collectif nécessite l'information et la consultation des représentants des salariées avant qu'une nouvelle renégociation ait lieu.

Les contrôleurs ne font pas que sanctionner le non-respect des règles, ils exercent aussi un pouvoir discrétionnaire pour laisser le temps aux partenaires sociaux de réviser leur texte. Comme l'indiquent les instructions de la DGT, la Direccte a également pour mission d'accompagner les entreprises vers la mise en œuvre de textes qui traitent de l'égalité professionnelle.

Le dispositif juridique établi par le législateur a donc permis de stimuler la négociation entre les parties en offrant un cadre propice à la mise en place de dispositions visant à résorber les inégalités professionnelles. Les mesures adoptées par les partenaires sociaux sont élaborées de telle façon qu'elles traduisent leur engagement dans la thématique.

\section{Un engagement paralysé par des prescriptions législatives}

Les textes sur l'égalité professionnelle visent à mettre en scène l'engagement des partenaires sociaux par une représentation articulée de mesures spécifiques. Alors que cet engagement s'inscrit dans un dispositif juridique qui structure son articulation, étudier les actions, véritable cœur des textes, permet d'apprécier leur contenu sans se limiter à l'observation du respect des contraintes juridiques.

\section{Le choix des domaines : un exercice réalisé entre la contrainte et le conformisme}

Le choix des domaines d'action, selon la nature juridique et la taille des entreprises, constitue le premier élément permettant de déterminer les différents axes d'engagement inscrits dans les textes sur l'égalité professionnelle.

17. Comme cela a été confirmé lors de l'entretien avec le contrôleur de la Direccte Gironde le 17 octobre 2017. 
Malgré l'opportunité de choix laissée aux négociateurs entre huit domaines d'action (avec l'obligation d'intégrer la rémunération), l'analyse des textes montre une convergence vers quatre domaines principaux : embauche, rémunération, formation et articulation $^{18}$, présents dans plus de la moitié des textes des deux échantillons ${ }^{19}$. Les négociations s'orientent donc vers des axes d'intervention considérés comme prioritaires, soulignés également par le rapport du CONSEIL SUPÉRIEUR DE L'ÉGALITÉ PROFESSIONNELLE ENTRE LES FEMMES ET LES HOMMES (2014) qui mentionne les mêmes thématiques.

Le domaine d'action « embauche » est le plus fréquent dans les deux échantillons. Les actions sont souvent conçues de sorte à prendre la forme d'une externalisation des responsabilités en matière de recrutement. Il s'agit de sensibiliser aux enjeux de mixité les partenaires extérieurs, tels que les agences d'intérim ou les établissements scolaires, pour qu'ils proposent d'eux-mêmes une « mixité » dans les candidatures ${ }^{20}$.

Dans les domaines «embauche », « rémunération » et «formation», les actions se caractérisent souvent par une simple formalisation des pratiques déjà présentes dans l'entreprise ou par des rappels du droit existant, dont l'adoption est simple et à faible coût, telles la formulation d'offres d'emploi avec l'en-tête «Recherche femme/homme » ou l'instauration d'un entretien annuel d'évaluation des formations suivies par les salarié.es.

La différence la plus nette concerne la fréquence de choix du domaine « articulation », avec un écart de 30 points entre les entreprises les plus grandes et les plus petites (tableau 4), et une fréquence plus élevée dans les accords que dans les plans d'action dans l'ensemble des textes. Les plus grandes entreprises affichent la volonté de mettre en avant l'articulation des temps de vie, dont les mesures (comme les dispositifs de télétravail et de formation en e-learning) sont potentiellement plus innovantes. Cette tendance s'explique par leur dotation importante en matière de personnel de ressources humaines, de compétences juridiques et de moyens financiers, leur permettant d'aboutir à des accords plus structurés que les petites entreprises.

Si l'obligation juridique invite à intervenir parmi neuf domaines d'action, les textes illustrent une tendance à marginaliser certains d'entre eux, plus complexes à traiter et dont les mesures à adopter risquent d'être particulièrement onéreuses.

On peut ainsi repérer un sous-ensemble de thématiques dont le choix demeure marginal. Il en va ainsi des domaines d'action «qualification » et «classification », thématiques relativement proches, absentes des textes analysés dans plus de $95 \%$ des cas. Ce constat doit toutefois être relativisé, car la classification professionnelle des salarié.es est un sujet abordé dans le cadre de négociations de branche laissant peu de marge de manœuvre aux entreprises.

\footnotetext{
18. L' « articulation entre l'activité professionnelle et la vie personnelle et familiale » est la dénomination précise du domaine d'action prenant en compte les temps de vie des salarié·es, mais par facilité d'usage, nous utiliserons le terme « articulation » dans notre développement.

19. Une exception concerne le domaine « articulation » qui n'est choisi qu'à hauteur de $45 \%$ parmi les plans des T. -300 .

20. Les rhétoriques le plus souvent mobilisées indiquent des difficultés à trouver, même au sein des établissements scolaires ou parmi les intérimaires, des candidats du sexe sous-représenté pour certains postes.
} 
TABLEAU 4 - Présence des domaines d'action par type de texte

\begin{tabular}{|c|c|c|c|c|c|c|}
\hline Domaines & $\mathbf{T}^{*} \cdot-300$ & T. 300+ & $\mathbf{P}^{*} \cdot-300$ & $A^{*} \cdot-300$ & P. 300+ & A. 300+ \\
\hline Embauche & 81 & 87 & 78 & 85 & 94 & 84 \\
\hline Formation & 59 & 72 & 55 & 65 & 80 & 69 \\
\hline Promotion & 34 & 55 & 21 & 50 & 40 & 60 \\
\hline Qualification & 6 & 1 & 8 & 5 & 3 & 0 \\
\hline Classification & 4 & 6 & 1 & 8 & 11 & 4 \\
\hline Conditions de travail & 26 & 20 & 33 & 20 & 17 & 21 \\
\hline Rémunération & 76 & 67 & 88 & 73 & 66 & 67 \\
\hline Articulation & 51 & 81 & 45 & 58 & 74 & 83 \\
\hline Nombre de textes & 140 & 142 & 74 & 66 & 35 & 107 \\
\hline
\end{tabular}

* T. : texte, P. : plan, A. : accord.

Lecture : le domaine d'action « embauche » est présent dans $78 \%$ des plans d'action des entreprises de moins de 300 salarié.es.

Champ : 282 textes négociés et signés entre 2011 et 2015 dans l'ancienne région Aquitaine (140 pour les T. -300 et 142 pour les T. 300+).

Source : données du projet Egapro.

La juxtaposition des thématiques proposées dans le dispositif sur l'égalité professionnelle avec d'autres thèmes de négociation obligatoire est un élément à prendre en compte. En effet, cette multiplication de négociations sur des thèmes similaires peut constituer un frein à l'engagement des acteurs de la négociation, amenés à traiter d'un même sujet à plusieurs reprises dans le cadre de négociations différentes imposées par le législateur. Il y a donc un risque de « boulimie bureaucratique », émanant de la volonté du législateur de structurer les thématiques au sein des différentes négociations, sans trop se soucier des répétitions des mêmes sujets, même abordés selon des perspectives différentes. C'est le cas du domaine obligatoire de la rémunération, dont le faible développement au sein des textes peut être la conséquence de son traitement dans une autre négociation, celle sur les salaires.

La volonté du législateur de lancer une négociation transversale sur plusieurs thématiques en obligeant les acteurs à négocier, par exemple, sur l'égalité professionnelle et la qualité de vie au travail risque également d'avoir un effet inverse et de limiter l'engagement sur la thématique de l'égalité, « noyée » dans d'autres sujets.

Cette incitation institutionnelle à négocier en cumulant les thématiques obligatoires a également une incidence sur la forme et sur la pertinence des mesures adoptées par les signataires. Certains domaines sont marginalisés, et les mesures adoptées ne font que formaliser des pratiques déjà appliquées dans l'entreprise (cf. supra) ou rappeler le droit existant. Les textes mentionnent tout élément en lien avec la thématique, mais n'élaborent pas nécessairement une stratégie qui permettrait de faire évoluer la situation.

L'éventail des domaines énoncés par la loi n'exclut pas la possibilité de développer d'autres thématiques lors des négociations, comme celles liées au secteur d'activité. Toutefois, le cadre imposé aux négociateurs réduit le recours à cette option. Seulement $6,3 \%$ des textes adoptent des mesures dans des domaines d'action non déjà listés par le législateur, ils sont alors souvent liés à la sensibilisation ou à la prévention du harcèlement sexuel au travail. 


\section{La structure de l'engagement : un cadre précis pour des mesures souvent de façade}

L'articulation tripartite des domaines d'action fixée par le législateur (objectifs, actions et indicateurs) contribue également aux différentes formes d'engagement exprimées dans les textes. Deux domaines d'action sont à cet égard particulièrement intéressants : la « rémunération » et l' « articulation ». Le premier en raison de l'obligation d'aborder cette thématique depuis la réforme de décembre 2012 et de son rôle dans la constitution des inégalités et des discriminations salariales. Le second du fait de son rayon d'action particulièrement étendu qui permet de réduire les inégalités entre les femmes et les hommes tout en développant une politique d'égalité en faveur de l'ensemble des salarié.es.

Afin de fixer une orientation aux mesures prises dans chaque domaine d'action, le législateur a exigé la présence d'au moins un objectif. Cela se traduit fréquemment par le choix de plusieurs objectifs, généraux ou difficilement identifiables. La multiplication des objectifs fixés par domaine d'action témoigne de la volonté des acteurs de la négociation de s'engager explicitement dans une diversité d'axes d'intervention. Dans le domaine "rémunération », peu d'objectifs sont généralement fixés puisque le nombre moyen y dépasse de peu l'unité obligatoire $(1,35)$. À l'opposé, le domaine « articulation » se rapproche d'une moyenne de deux objectifs. Cette différence s'explique par la divergence des finalités et de leurs spécificités. Alors que les objectifs en matière de rémunération sont concentrés sur la résorption des écarts salariaux, les visées en matière d'articulation des temps de vie sont plus diverses et prennent en compte le temps partiel, les congés parentaux ou encore l'organisation des réunions de travail.

Une fois les objectifs de chaque domaine fixés, le dispositif juridique exige qu'une ou plusieurs actions précisent les moyens de les atteindre. Elles constituent l'élément central de l'articulation du dispositif, car elles représentent les formes concrètes d'engagement exprimées dans les textes. Leur nombre souligne le niveau d'intérêt et de mobilisation des partenaires sociaux pour une thématique particulière, et leur contenu permet d'apprécier les moyens humains, techniques et financiers mis en œuvre pour les appliquer et ainsi faire évoluer la situation dans l'entreprise. Les actions sont en moyenne plus nombreuses que les objectifs, mais le manque de précision de certaines mesures se traduit par une fusion de la description des objectifs et des actions.

Dans la plupart des domaines, on compte en moyenne entre 2,5 et un peu plus de 3 actions avec, une nouvelle fois, un écart entre les domaines « rémunération » et « articulation» (tableau 5). C'est dans le domaine « rémunération » qu'il y a en moyenne le moins d'actions dans les différentes catégories de textes, particulièrement dans les T. 300+ où les plans d'action comprennent moins de deux actions. Dans le domaine « articulation », il y a en moyenne plus d'actions $(4,5)$ dans les T. 300+, la variété des actions témoignant d'une plus grande innovation. Certains domaines sont choisis et argumentés au minimum pour se conformer à l'obligation légale, alors que 
TABLEAU 5 - Nombre moyen d'actions pour les domaines d'action « rémunération » et « articulation » selon le type de texte

\begin{tabular}{lcccccc}
\hline Domaines d'action & $\mathbf{T}^{*} . \mathbf{- 3 0 0}$ & T. 300+ & $\mathbf{P}^{*}$. $\mathbf{- 3 0 0}$ & $\mathbf{A}^{*}$. $-\mathbf{3 0 0}$ & $\mathbf{P . 3 0 0 +}$ & A. 300+ \\
\hline Rémunération & 2 & 2,1 & 2 & 2,1 & 1,8 & 2,2 \\
Articulation & 3,2 & 4,5 & 3,2 & 3,1 & 3,5 & 4,7 \\
\hline Nombre de textes & $\mathbf{1 4 0}$ & $\mathbf{1 4 2}$ & $\mathbf{7 4}$ & $\mathbf{6 6}$ & $\mathbf{3 5}$ & $\mathbf{1 0 7}$ \\
\hline
\end{tabular}

* T. : texte, P. : plan, A. : accord.

Lecture : on compte en moyenne deux actions pour le domaine « rémunération » pour les entreprises de moins de 300 salarié $\cdot e s$. Champ : 282 textes négociés et signés entre 2011 et 2015 dans l'ancienne région Aquitaine (140 pour les T. - 300 et 142 pour les T. 300+). Source : données du projet Egapro.

d'autres font l'objet d'un descriptif détaillé. La polarisation entre les domaines « rémunération » et «articulation » montre une structuration différente des actions selon qu'elles sont imposées par le législateur ou qu'elles résultent de la libre expression des négociateurs. Si les textes suivent l'obligation de s'impliquer dans la « rémunération », dans la plupart des cas, ce respect ne se traduit pas par un effort notable dans l'engagement. A contrario, le domaine « articulation », bien que moins fréquemment choisi, est plus travaillé et présente plus de mesures susceptibles d'avoir des effets concrets sur la vie de l'entreprise.

Le législateur exige également que l'entreprise se dote d'indicateurs chiffrés pour suivre les objectifs et les actions dans les textes, et pour détailler, par des objectifs chiffrés et des procédures de suivi, comment sera évaluée l'efficacité des mesures souscrites. L'analyse des données fait état d'un nombre réduit d'indicateurs par rapport au volume des actions.

C'est de nouveau dans le domaine d'action « rémunération» que les moyennes sont les plus basses et dans celui de l' « articulation » qu'elles sont les plus élevées. Au sein de la thématique « rémunération », les indicateurs ne sont présents que dans $60 \%$ des textes. Un tel constat confirme que l'engagement dans ce domaine obligatoire est limité : les mesures n'y sont pas articulées de façon concrète, mais semblent seulement répondre aux injonctions législatives.

La faible présence d'indicateurs au sein des textes souligne les difficultés que rencontrent les acteurs de la négociation à prévoir et à instituer des formes d'évaluation et de contrôle des mesures souscrites. La présence plus fréquente d'indicateurs parmi les T. 300+ confirme toutefois la meilleure articulation des mesures formulées par les entreprises ayant, du fait de leur grande taille, une organisation plus structurée. Ces résultats sont comparables à ceux du rapport pour la Direccte Alsace (BUCHER et al., 2015), qui pointait également le manque d'indicateurs associés aux domaines d'action $^{21}$.

21. Le nombre moyen d'actions par domaine y est néanmoins différent : 1,7 pour la rémunération (contre environ 2 dans nos deux échantillons) et 2,8 pour l'articulation (BUCHER et al. 2015, p. 30), ce qui est sensiblement moins que les chiffres calculés en Aquitaine (3,2 parmi les T. -300 et 4,5 dans les T. 300+). 
L'obligation introduite par le législateur pour obtenir une structuration «modèle » des textes a été largement suivie (à l'exception, donc, des indicateurs et des difficultés qu'ils posent), mais n'a rien prévu quant au contenu des mesures, qui demeure hors de portée des contrôles.

\section{Les actions, cœur de l'engagement ou coquilles vides?}

L'action est l'élément clé du dispositif dont l'analyse permet d'apprécier les modalités d'engagement exprimées dans les textes pour atteindre les objectifs fixés dans chaque domaine d'action. Nous avons donc établi une typologie ${ }^{22}$ (encadré 2) pour détailler la forme, le contenu, les destinataires et les personnes chargées de la mise en œuvre des 2986 actions dénombrées ${ }^{23}$. Cette typologie vise à différencier les formes d'engagement détaillées dans les actions afin d'indiquer les modalités d'intervention au sein des entreprises en proposant des étiquettes qui font référence à un répertoire d'actions ayant des éléments en commun.

La répartition des actions selon notre typologie (tableau 6) révèle de manière flagrante que les actions «non descriptives » $(28 \%$ du total) sont plus fréquemment formulées que celles relatives à l' « aménagement » $(24 \%)$ et à la « proportionnalité » $(16 \%)$.

TABLEAU 6 - Nombre et part des actions selon la typologie établie

\begin{tabular}{lcc}
\hline Type d'action & Effectif & Part (\%) \\
\hline Non descriptive & 840 & 28 \\
Aménagement & 708 & 24 \\
Évaluation & 318 & 11 \\
Information/communication & 283 & 9 \\
Proportionnalité & 485 & 16 \\
Sensibilisation & 352 & 12 \\
\hline Total & $\mathbf{2 9 8 6}$ & $\mathbf{1 0 0}$ \\
\hline
\end{tabular}

Lecture : sur les 2986 actions recensées, 840, soit $28 \%$, sont des actions non descriptives.

Champ : 2986 actions contenues dans les 282 textes négociés et signés entre 2011 et 2015 dans l'ancienne région Aquitaine (140 pour les T. -300 et 142 pour les T. $300+$ ).

Source : données du projet Egapro.

22. Cette typologie des actions se différencie en partie de celle réalisée par les auteurs du rapport pour la Direccte Alsace (Bucher et al., 2015), qui est également constituée de six classes d'actions dont une résiduelle (« autres »). Les différences se situent dans la définition des actions les plus concrètes. Alors que nous parlons de « sensibilisation/ accompagnement » et d' « aménagement », les auteurs font référence à l' « augmentation de l'offre » et à l' « incitation ». Une autre divergence réside dans la définition des actions « non descriptives » : alors que nous avons établi des critères pour les isoler dès le départ tout en essayant de minimiser l'appréciation subjective, le rapport pour la Direccte Alsace présente des indicateurs subjectifs relatifs à la cohérence pour évaluer les engagements « d'une part sur la présence de modalités concrètes de mise en œuvre, d'autre part sur la cohérence entre l'action, l'objectif assigné et l'indicateur de suivi retenu et sur la pertinence globale de l'action » (BUCHER et al. 2015, p. 3).

23. Nous avons recensé 2986 actions réparties au sein de quatre domaines d'action principaux, représentant $80 \%$ de l'ensemble des actions : «embauche » $(26 \%)$, « articulation » $(24 \%)$, «formation » $(17 \%)$ et « rémunération » $(14 \%)$. 
ENCADRÉ 2

\section{Typologie du contenu des actions}

\section{Information}

Actions présentant des informations factuelles avec ou non des destinataires explicites. Exemples : réalisation d'affiches, campagnes de promotion et de publicité, journées de sensibilisation, etc.

\section{2. Évaluation}

Actions proposant une analyse chiffrée de l'entreprise pour identifier et/ou évaluer une problématique ou mener une investigation.

Exemples : réalisation d'enquêtes, rapports, bases de données, diagnostics, etc.

\section{Sensibilisation}

Actions détaillant la façon dont l'entreprise prévoit de sensibiliser et/ou d'accompagner les salariées sur une thématique, une problématique ou dans l'application ou le suivi d'une procédure ou d'une règle.

Exemples : relations entre acteurs, parcours d'intégration et incitation à participer à une formation spécifique, parrainage, etc.

\section{Aménagement}

Actions dont le contenu indique une modification, une possibilité supplémentaire, un changement dans les conditions de travail.

Exemples : journées enfant malade, réduction de la pénibilité, pas de réunions tardives, etc.

\section{Proportionnalité}

Actions dont le contenu décrit les modalités d'accès ou de mise en œuvre de mesures et précise les critères à appliquer.

Exemples : au moins un homme/une femme auditionnée pour chaque poste, etc.

\section{Non descriptive}

Actions n'ayant pas de contenu articulé, ne présentant pas d'objet clair ni de plan d'application. Rédigées de manière floue ou se contentant d'énoncer des règles de droit.

Exemples : "Diversifier les sources de recrutement ", "Maintenir un lien avec le référent Pôle emploi », "Porter une attention particulière à la situation des familles monoparentales », etc.

Cette typologie permet d'apprécier les modalités de l'engagement dans les différents domaines. La catégorie d'action « non descriptive » met en exergue les mesures dont le contenu n'est ni articulé ni vraiment identifiable. Une forte proportion d'actions « non descriptives » signale un texte faiblement incitatif et dont il est possible de douter de l'efficacité. 
Ce type de textes ressemble fréquemment à des « coquilles vides » (CHARPENEL et al., 2017 ; HoQue, NoOn, 2004). S'il est difficile d'apprécier précisément l'ampleur du phénomène au sein de textes si hétérogènes, la forte fréquence d'actions non descriptives est le symptôme du manque de caractère concret d'un texte. Plus de la moitié des actions formulées dans $16,7 \%$ de l'ensemble des textes sont « non descriptives », ce qui illustre les difficultés à atteindre l'objectif de « mesures concrètes pour faire évoluer la situation », comme cela est énoncé par la communication gouvernementale. Les actions «non descriptives » demeurent plus nombreuses parmi les textes des plus petites entreprises ( $26,6 \%$ contre seulement $7 \%$ pour les grandes), ce qui est le signe de leurs difficultés à élaborer des mesures concrètes, comme le confirme la faible présence des actions classées comme « aménagement » dans ces mêmes petites entreprises ( $16 \%$ dans les T. -300 et $29 \%$ parmi les T. $300+$ ).

La répartition par types d'action permet d'apprécier les modalités d'engagement de chaque domaine d'action. Ainsi, le domaine « articulation » se distingue par la forte part d'actions ayant un caractère concret, en particulier du type « aménagement » $(50 \%)$, et par la part d'actions « non descriptives » la plus faible de tous les domaines $(15 \%)$. La question de l'articulation des temps de vie est en effet une thématique sensible pour l'ensemble des salarié-es, et particulièrement pour les chargé es de famille pour lesquel-les cela constitue un facteur important dans leur perception du bien-être au travail (HoBson, 2013).

Dans le domaine « rémunération », les actions d' «évaluation » sont présentes dans un tiers des cas (34\%) tandis que celles qualifiées de « non descriptives » représentent un autre tiers (32\%). Dans ce domaine obligatoire, les actions d' " évaluation », qui mobilisent les chiffres de l'entreprise (issus du rapport de situation comparée ou de la base de données économiques et sociales), sont fréquentes, car elles ont pour objectif de clarifier les modalités de rémunération et de déterminer des critères d'évolution des salaires. Les nombreuses actions «non descriptives » confirment, quant à elles, les difficultés rencontrées par les acteurs de la négociation pour rédiger des diagnostics chiffrés sur la situation de l'entreprise. Encore une fois, le domaine imposé par le législateur apparait comme le moins articulé, ce qui est peut-être le signe d'une résistance des acteurs à consacrer des efforts au-delà du seul respect formel de l'obligation ou de la volonté de ne pas communiquer clairement sur cette question.

La présence d'un investissement financier de l'entreprise est un autre élément clé pour attester de la volonté d'accomplissement d'un engagement. L'investissement financier permet le suivi des actions, mais l'information relative au budget consacré à l'application des mesures concerne seulement $3 \%$ des actions analysées. Cela peut être le signe d'une absence de volonté des employeurs de s'engager sur des mesures chiffrées financièrement.

Cette constatation est dans la lignée de ce que nous avons observé de manière générale, c'est-à-dire une réticence de la part des acteurs de la négociation à s'engager à fournir des indications concrètes et chiffrées concernant l'adoption et le suivi des actions. La relative fragilité de l'engagement se traduit aussi dans la tendance à répéter les mêmes mesures lors des renégociations des textes. Nous avons ainsi analysé deux 
textes issus d'une même entreprise et de deux négociations successives ; nous avons compté les répétitions et quantifié les « soupçons de copier-coller » ${ }^{24}$ afin d'évaluer l'ampleur de la pratique qui consiste à négocier dans l'objectif du seul respect formel des obligations et à prendre des engagements de « façade » sans réel objectif de progression. De fait, un tiers des entreprises ayant renégocié présentent des textes avec une structure, des domaines d'action et un nombre d'éléments qui sont, dans la majorité des cas, très semblables, voire identiques, aux versions antérieures.

Les éléments mis en lumière montrent comment l'engagement exprimé dans les textes est souvent plus orienté vers le respect formel des obligations et présente donc de nombreuses limites quant à sa capacité d'articuler des mesures claires dans une optique de continuité et de progression.

Les inégalités professionnelles entre les femmes et les hommes sont enracinées dans le quotidien des entreprises (MARUANI, 1998) et ne peuvent se résorber, ou s'atténuer, que si les engagements sont pris et tenus de façon continue. Parce qu'évaluer et suivre les dysfonctionnements en respectant les périodes de couverture des textes n'est pas chose aisée, s'intéresser à la logique diachronique de l'engagement exprimé dans ces derniers permet d'évaluer l'efficacité des actions formulées et de relever l'existence d'une politique de progression. Cette analyse, menée ici sur cinq années, a permis de mettre en exergue certains éléments.

D'abord, le fait que de nombreux textes renégociés ne fassent aucune référence aux mesures précédemment souscrites démontre l'absence de continuité dans les renégociations. Ensuite, la confrontation de textes successifs fait apparaitre des contenus identiques dans le choix des domaines d'action ou des mesures permettant d'articuler leur contenu. D'où une interrogation sur la qualité de la négociation, qui ressemble plus à un respect formel des obligations qu'à un engagement concret en faveur de l'égalité professionnelle. Enfin, l'absence de suivi des indicateurs et des références aux axes et aux mesures précédemment souscrites suggère une absence de prise en compte des textes précédents. Ce manque d'indications sur les engagements pris par le passé dans les textes renégociés laisse penser que les engagements sont purement formels, sans logique de progression ni de suivi des actions (FREYSSINET, 2010).

L'analyse des textes renégociés montre par ailleurs comment les interventions législatives sur la thématique de l'égalité professionnelle ont eu pour effet d'augmenter la négociation, qui demeure toutefois superficielle et conformiste et qui témoigne rarement d'un véritable effort dans la co-construction entre partenaires sociaux d'une dynamique

24. Nous avons pris en compte les domaines d'action et le nombre total d'objectifs, d'actions et d'indicateurs dans l'ensemble des domaines choisis. Après avoir effectué une confrontation des textes de la même entreprise, nous avons calculé un indice de « similitude » compris entre 1 et 10 indiquant un soupçon de « copier-coller » à partir des correspondances et des écarts entre les textes. Nous parlons de « soupçon », car seule une confrontation intégrale du contenu des textes, hors de notre portée, aurait pu expliciter le degré effectif de « copier-coller». 
de progression dans la réduction des inégalités. Or, de ce point de vue, un changement ne pourra être effectif qu'à la condition de concentrer les efforts, et donc les contrôles, sur le contenu des textes plutôt que sur le respect formel de l'obligation. Cela ne pourra se faire sans une démarche de suivi, ponctuel et chiffré, qui serait un levier pour élaborer des mesures spécifiques, et non se contenter d'engagements sur «papier».

Les interventions législatives successives n'ont pas permis jusqu'à présent d'obtenir les résultats escomptés : la contrainte demeure insuffisante (un grand nombre d'entreprises ne sont pas couvertes par un accord ou un plan d'action), les textes signés demeurent très formels, et le suivi des mesures, précaire. La multiplication des obligations sur une thématique pourrait avoir pour effet pervers de conduire les acteurs de la négociation à se concentrer sur le seul respect des critères juridiques. Les potentielles réductions des inégalités paraissent donc limitées par un dispositif dépourvu d'obligations de résultat. C'est la raison pour laquelle le législateur est intervenu dans le cadre de la loi sur la liberté de choisir son avenir professionnel du 5 septembre 2018 pour instituer une obligation de résultat concernant les écarts de rémunération entre les femmes et les hommes. La loi oblige désormais les entreprises à publier, sous peine de se voir appliquer une sanction financière, un index sur les écarts de salaire et prévoit la mise en place de mesures de correction et de rattrapage salarial en appliquant le dispositif de négociation sur l'égalité professionnelle. Les entreprises sont contraintes d'obtenir et de publier cet index au risque de "dégrader » leur image. Dès lors qu'il existe des écarts de rémunération persistants, l'entreprise a l'obligation de les résorber par la négociation sur l'égalité professionnelle, qui devient un outil de mise en œuvre de cette nouvelle obligation. Cette nouvelle obligation de résultat devrait donc inciter les entreprises à négocier et à permettre ainsi de produire des accords ou des plans d'action adaptés au contexte spécifique de chaque entreprise, petite ou grande.

\section{BibLIOGRAPHIE}

AMINTAS A., JUnTER A. (2009), «L'égalité prise au piège de la rhétorique managériale », Cahiers du genre, $\mathrm{n}^{\circ} 47$, pp. 103-122.

BRunet S., Dumas M. (2012), « Bilan de l'application des dispositifs promouvant l'égalité professionnelle entre femmes et hommes », Les Études du Conseil, économique, social et environnemental, $\mathrm{n}^{\circ} 7$.

Bucher A., Forté M., Garat T., Moizard N., Terraz I., Tournadre F. (2015), Négocier l'égalité professionnelle entre les femmes et les hommes : avancées, résistances et conditions de développement, rapport de recherche pour la Direccte Alsace, université de Strasbourg.

BUREAU INTERNATIONAL DU TRAVAIL (2012), Égalité entre hommes et femmes et travail décent. Conventions et recommandations clés de l'OIT pour la promotion de l'égalité entre hommes et femmes. 2012, Genève, Organisation internationale du travail.

Charpenel M., Demilly H., Pochic S. (2017), «Égalité négociée, égalité standardisée ? », Travail, genre et sociétés, ${ }^{\circ}$ 37, pp. 143-147. 
Commaille J. (2001), « Les injonctions contradictoires des politiques publiques à l'égard des femmes », in Laufer J., Marry C., Maruani M. (dir.), Masculin-Féminin : questions pour les sciences de l'homme, Paris, Presses universitaires de France, pp. 129-148.

Commaille J., Duran P. (2009), « Pour une sociologie politique du droit : présentation », L’Année sociologique, vol. ${ }^{\circ}$ 59, pp. 11-28.

CONSEIL SUPÉRIEUR DE L'ÉGALITÉ PROFESSIONNELLE ENTRE LES FEMMES ET LES HOMMES (2014), La Négociation collective sur l'égalité professionnelle dans les entreprises de 50 à 300 salariés en 2012 et 2013, rapport du Conseil supérieur de l'égalité professionnelle entre les femmes et les hommes, Paris.

CRistofalo P. (2014), « Négocier l'égalité professionnelle : de quelques obstacles à la prise en charge syndicale de la thématique », Nouvelle revue de psychosociologie, ${ }^{\circ}$ 18, pp. 133-146.

DAuphin S. (2011), « Action publique et rapports de genre », in Milewski F., Périvier H. (dir.), Les Discriminations entre les femmes et les hommes, Paris, Presses de Sciences Po, pp. 313-341.

Delpeuch T., Dumoulin L., Galembert C. (DE) (2014), Sociologie du droit et de la justice, Paris, Armand Colin.

DireCCTE PACA (2013), « Analyse d'une sélection d'accords et de plans d'action d'entreprise signés en Provence-Alpes-Côte d'Azur sur l'égalité professionnelle entre les hommes et les femmes : des accords conformes mais perfectibles », Théma, $\mathrm{n}^{\circ} 36$.

Dugué B. (2005), Le Travail de négociation. Regards sur la négociation collective d'entreprise, Toulouse, Octarès.

FREYSSINET J. (2010), « Les négociations collectives et les politiques publiques face aux "conséquences sociales" de la crise économique », Revue de l'OFCE, n 115, pp. 81-120.

Groux G. (2005), «L'action publique négociée. Un nouveau mode de régulation ? Pour une sociologie politique de la négociation », Négociations, ${ }^{\circ} 3$, pp. 57-70.

Hassenteufel P. (2011), Sociologie politique : l'action publique, $2^{\mathrm{e}}$ éd. revue et enrichie, Paris, Armand Colin.

Hobson B. (ed.) (2013), Worklife Balance: The Agency and Capabilities Gap, Oxford, Oxford University Press.

Hoque K., Noon M. (2004), "Equal Opportunities Policy and Practice in Britain: Evaluating the 'Empty Shell' Hypothesis”, Work, Employment and Society, vol. 18, n 3, pp. 481-506.

Jobert B., Muller P. (1987), L'État en action. Politiques publiques et corporatismes, Paris, Presses universitaires de France.

JUNTER A. (2004), «L'égalité professionnelle entre les femmes et les hommes : une exigence politique au cœur du droit du travail », Travail, genre et sociétés, $\mathrm{n}^{\circ} 12$, pp. 191-202.

JUNTER-LOISEAU A. (1996), L'Égalité professionnelle dans la négociation collective, rapport pour la Fondation de Dublin pour l'amélioration des conditions de vie et de travail.

LANQUETIN M.-T. (2017), « Du droit européen au droit français : comment parvenir à l'égalité en fait et en droit ?», Travail, genre et sociétés, n³ 37, pp. 137-141. 
Lanquetin M.-T., Letablier M.-T., Périvier H. (2004), « Acquisition des droits sociaux et égalité entre les femmes et les hommes », Revue de l’OFCE, n 90, pp. 461-488.

LAscoumes P., Le Bourhis J.-P. (1996), « Des “passe-droits” aux passes du droit. La mise en œuvre socio-juridique de l'action publique », Droit et Société, n 32, pp. 51-73.

LAUFER J. (2009), « L'égalité professionnelle entre les hommes et les femmes est-elle soluble dans la diversité ?», Travail, genre et sociétés, $\mathrm{n}^{\circ} 21$, pp. 29-54.

LAUFER J. (2014), L'Égalité professionnelle entre les femmes et les hommes, Paris, La Découverte.

Laufer J., Silvera R. (2005), Accords sur l'égalité professionnelle suite à la loi du 9 mai 2001 : premiers éléments d'analyse. Projet TIMETIS, l'égalité hommes/femmes dans la vie personnelle et professionnelle, Montreuil, Émergences.

Laufer J., Silvera R. (2017), « Controverse. Des lois à la négociation... Quoi de neuf pour l'égalité professionnelle ? Présentation », Travail, genre et sociétés, n 37, pp. 129-132.

Le Quentrec Y., BACOU M. (2017), « La négociation collective de l'égalité professionnelle : une mise en œuvre sous tensions », Travail, genre et sociétés, n 37, pp. 149-153.

MARUANi M. (dir.) (1998), Les Nouvelles Frontières de l'inégalité : hommes et femmes sur le marché du travail, Paris, La Découverte.

MARUANi M. (dir.) (2013), Travail et genre dans le monde, l'état des savoirs, Paris, La Découverte.

Mias A. (2014), «Entre complexification et simplification du travail de négociation. L'ambivalence des pratiques de connaissance en entreprise », Nouvelle revue de psychosociologie, $\mathrm{n}^{\circ} 18$, pp. 41-53.

Milewski F., SÉnAC R. (2014), « L'égalité femmes-hommes. Un défi européen au croisement de l'économique, du juridique et du politique », Revue de l'OFCE, n 134, pp. 201-213.

Miné M. (2017), « La négociation de l'égalité à l'épreuve des réformes du Code du travail », Travail, genre et sociétés, $\mathrm{n}^{\circ} 37$, pp. 133-136.

Ministère dU TravaIL (2018), La Négociation collective en 2017. Édition 2018, Paris, ministère du Travail.

Padioleau J.-G. (1982), L'État au concret, Paris, Presses universitaires de France.

PfEFFERKORN R. (2002), « Les politiques publiques et la question de l'égalité hommes-femmes. Le cas de la France », Cahiers du genre, n 32, pp. 87-109.

RABIER M. (2009), « Analyse du contenu des accords d'entreprise portant sur l'égalité professionnelle entre les femmes et les hommes signés depuis la loi du 23 mars 2006 », in Ministère du Travail, La Négociation collective en 2008, Paris, ministère du Travail, pp. 423-459.

SANTORO G. (2016), « Réflexions sur l'efficacité du droit de la négociation collective sur l'égalité hommes-femmes », Droit social, $\mathrm{n}^{\circ} 1$, pp. 49-57.

Silvera R. (2006), «Le défi de l'égalité hommes/femmes dans le syndicalisme », Mouvements, n 43 , pp. 23-29.

WELLER J.-M. (2007), « La disparition des bœufs du père Verdon. Travail administratif ordinaire et statut de la qualification », Droit et Société, $\mathrm{n}^{\circ}$ 67, pp. 713-735. 